\title{
LncRNA MALAT1 promotes neuropathic pain progression through the miR-154-5p/AQP9 axis in CCI rat models
}

\author{
JIANPING WU, CHUANGUANG WANG and HAIYANG DING \\ Department of Anesthesia, Lishui Municipal Central Hospital, Lishui, Zhejiang 323000, P.R. China
}

Received March 9, 2019; Accepted October 15, 2019

DOI: $10.3892 / \mathrm{mmr} .2019 .10829$

\begin{abstract}
The present study investigated the role and molecular mechanism of long non-coding RNA (lncRNA) metastasis associated lung adenocarcinoma transcript (MALAT)1 in neuropathic pain in rat chronic constriction injury (CCI) model. Reverse transcription-quantitative PCR and western blot analysis were used to detect the expression levels of MALAT1, microRNA (miR)-154-5p and aquaporin (AQP) 9 in spinal cord tissue and microglia of CCI rats. ELISA and pain behavioral assays were used to observe the effect of MALAT1 on neuropathic pain and neuroinflammation in model rats, and to verify its molecular mechanism through bioinformatics and luciferase experiments. The results of the present study identified that the expression levels of MALAT1 and AQP9 were upregulated, while miR-154-5p was downregulated in spinal cord tissue and microglia of CCI rats. MALAT1 knockdown in CCI model rats significantly induced the occurrence of neuropathic pain, while the upregulation of miR-154-5p could reverse this process. The present study also identified that miR-154-5p was the target gene of MALAT1, and AQP9 was the target gene of miR-154-5p. AQP9 knockdown promoted the occurrence of neuropathic pain. In conclusion, IncRNA MALAT1 promotes the progression of neuropathic pain in rats by reducing miR-154-5p and increasing AQP9. The MALAT1/miR-154-5p/AQP9 axis can be used as a new therapeutic target for neuropathic pain.
\end{abstract}

\section{Introduction}

Neuropathic pain following spinal surgery is a problem that has the common characteristics of being severe and chronic for patients (1-3). Although much progress has been made in clinical treatment, a number of patients still suffer chronic pain and psychological problems (4). At present, the molecular

Correspondence to: Dr Haiyang Ding, Department of Anesthesia, Lishui Municipal Central Hospital, 289 Kuocang Road, Lishui, Zhejiang 323000, P.R. China

E-mail: ding_haiyang2010@163.com

Key words: IncRNA MALAT1, miR-154-5p/AQP9 axis, CCI model, neuropathic pain mechanism of neuropathic pain remains unclear. To provide a new treatment for neuropathic pain, it is urgent to understand its molecular mechanism.

It has been reported that proinflammatory factors may participate in regulating pain sensitivity and inducing chronic pain by activating the secretion of inflammatory molecules such as interleukin (IL)- $1 \beta$, tumor necrosis factor (TNF)- $\alpha$, cyclooxygenase (COX)-2 and IL-6 (5). Therefore, targeting inflammation signaling pathways may provide potential therapeutic opportunities in neuropathic pain. It has been reported that enhancer of zeste homolog 2 (EZH2) signaling pathway could inhibit spinal neuroinflammation and alleviate occurrence and development of neuropathic pain (6).

Long non-coding RNAs (IncRNAs) are a type of RNA that are longer than 200 nucleotides but do not have capacity for encoding protein; they participate in a number of biological processes in various diseases $(7,8)$. It has been reported that lncRNAs can be involved in the regulation of several neurological diseases including spinal cord injury (SCI) (9). For example, lnc $\mathrm{X}$-inactive specific transcript can regulate the spinal cord injury in rats by responding to micro (miR)-494 (9). Metastasis associated lung adenocarcinoma transcript (MALAT)1 has been identified in multiple types of physiological and pathological processes, including organizing nuclear construction, modulating gene expression, participating in diabetes complications and in cancers (10-14). lnc-MALAT1 participates in the post-ischemic perfusion neuroprotection by interacting with miR-204 (15). However, whether it participate in neuropathic pain remains to be elucidated.

Several studies have reported that miRNAs are involved in the development of neuropathic pain. For example, miR-183 inhibits neuropathic pain by inhibiting the mTOR/vascular endothelial growth factor signaling pathway (16). It can activate p38 through downregulating miR-128 and then trigger neuropathic pain (17). The dysregulation of miR-154-5p is common in a number of tumors. For example, miRNA-154-5p inhibits the proliferation, migration and invasion of prostate cancer cells by targeting E2F transcription factor 5 (18) and miRNA-154-5p inhibits cell proliferation and metastasis by targeting Piwi like RNA-mediated gene silencing 4 (19). However, the role of miR-154-5p in neuropathic pain remains to be elucidated.

Aquaporin (AQP) 9 is a protein encoded by the AQP9 gene in humans, which belongs to the aquaporin family and is a type of water-selective membrane channel that allows the 
passage of multiple non-charged solutes, including water, glycerol, purine, pyrimidine and lactic acid. It serves a key role in leukocyte immune response and bactericidal activity $(20,21)$. AQP9 also serves an important role in tumor cells. For example, AQP9 can activate the Akt signaling pathway to promote astrocytoma cell invasion (22). It can also arrest cell cycle by inducing Ras to improve the efficacy of colorectal cancer chemotherapy (23). However, the role of AQP9 in neuropathic pain is unclear.

The present study attempted to investigate the role of MALAT1 in the development of neuropathic pain. A significant increase of MALAT1 expression in the chronic constriction injury (CCI) rat model was observed, whereas inhibition of MALAT1 attenuated the development of neuropathic pain. In a rat model of neuropathic pain, miR-154-5p was decreased, while AQP9 was increased. Therefore, it was speculated that MALAT1 could serve an important role in the development of neuropathic pain by regulating miR-154-5p/AQP9.

\section{Materials and methods}

Animal studies. A total of 60 adult SD rats (female, 4 weeks, 180-200 g) were purchased from Shanghai Animal Laboratory Center and randomly divided into sham control group and CCI groups. Rats were housed in standard plastic cages at $24 \pm 1^{\circ} \mathrm{C}$ and $50-70 \%$ of humidity. The rats were kept in the animal environment on a 12-h light-dark cycle and could drink and eat freely. In the CCI rat groups, the rats were divided into LV-NC, LV-shMALAT1, LV-miR-154-5p and LV-shAQP9 groups (12 rats in each group). In addition, miR-154-5p inhibitor or miR-NC was injected intrathecally into rats treated with LV-shMALAT1 for 3 days (4 rats in each group). Rats were group-fed in every cage at $25^{\circ} \mathrm{C}$. An animal model of neuropathic pain model was established by the CCI method (24). Rats were intraperitoneally injected with sodium pentobarbital (40 $\mathrm{mg} / \mathrm{kg}$ ) for anesthesia. In the CCI rat groups, the bilateral sciatic nerves of two legs were exposed and ligated, while nothing was ligated in the sham group. Then, 3 rats in each group were randomly selected and sacrificed following surgery for days $0,3,7$ and 14, the spinal cord tissue was collected and microglia were isolated for further study. Following CCI surgery, different recombinant lentivirus was respectively injected into the rats through intrathecal microneedle injection for lentiviral infection. The present study was carried out in strict accordance with the requirements in the Guide for the Care and Use of Laboratory Animals of the National Institutes of Health (25). The experiments were carried out strictly in line with the requirements of the International Association for the Study of Pain (IASP) (26). The present study was approved by the ethics committee of Lishui Municipal Central Hospital. In order to minimize the time that rats suffered pain from experimental treatment as much as possible, when the following pain characteristics were observed in each rat, they were rapidly euthanized by cervical dislocation during deep anesthesia at the end of the experiment: Rapid weight loss, self-mutilation, muscle stiffness, biting/aggression and skin dehydration.

Cell isolation and cell culture. Primary microglia were isolated according to the method of Beltramo et al (27). The spinal cord tissue from rats was collected and was digested by $0.25 \%$ trypsin at $4^{\circ} \mathrm{C}$ for 20 mins. Following centrifugation at $4^{\circ} \mathrm{C}$ and $800 \mathrm{x} \mathrm{g}$ for $5 \mathrm{~min}$, the mixed glial cells were isolated and cultured in DMEM/F12 medium (Invitrogen; Thermo Fisher Scientific, Inc.) containing 10\% fetal bovine serum (FBS; Gibco; Thermo Fisher Scientific, Inc.) at $37^{\circ} \mathrm{C}$ and $5 \% \mathrm{CO}_{2}$ culture incubator. After two weeks, microglia were isolated from the mixed glial cells and cultured in $\mathrm{DMEM} / \mathrm{F} 12$ medium containing $10 \% \mathrm{FBS}$ at $37^{\circ} \mathrm{C}$ and $5 \% \mathrm{CO}_{2}$ culture incubator.

Construction of lentivirus and cell transfection. The lentivirus vectors of LV-NC (cat. no. D03003, Shanghai GenePharma Co., Ltd.), LV-shMALAT1, LV-miR-154-5p and LV-shAQP9 were synthesized by Shanghai GenePharma Co, Ltd. Following CCI surgery, these lentivirus vectors $\left(1 \times 10^{7} / 0.1 \mathrm{ml}\right)$ were respectively injected into the rats through intrathecal microneedle injection for infection. Microglia cells were seeded in 6-well plates $\left(2 \times 10^{6} /\right.$ well) until reached $70-80 \%$, before transfection, the transfection reagent Lipofectamine ${ }^{\circledR}$ 2000 (Invitrogen; Thermo Fisher Scientific, Inc.), serum-free DMEM and $100 \mathrm{nM}$ miR-NC (cat. no. miR0190513015853, Guangzhou RiboBio Co., Ltd.) or 100 nM miR-154-5p mimics (cat. no. miR10000452-1-5, Guangzhou RiboBio Co., Ltd.) were mixed and incubated for $30 \mathrm{~min}$, and then added into microglia with complete medium containing $15 \%$ FBS. At the indicated time point following transfection, cells were harvested for further study. Relative expression levels of miR-154-5p were significantly increased in cells transfected with miR-154-5p mimics compared with in cells transfected with miR-NC (data not shown).

Detection of Cox-2, IL- 6 and TNF- $\alpha$ levels by ELISA. The spinal cord tissues were collected and the microglia isolated. Protein lysate was added to the spinal cord tissue and microglia samples of each group to homogenize the tissue, and the supernatant was collected following centrifugation with $8,000 \times \mathrm{g}$ at $4^{\circ} \mathrm{C}$. Levels of COX-2 (cat. no. kt22030, rat), IL-6 (cat. no. kt22084, rat) and TNF- $\alpha$ (cat. no. kt30484, rat) were detected by ELISA kits according to the manufacturer's protocol (Wuhan MSK Biotechnology Co, Ltd.). The concentrations of the standard wells were $0,7.5,15$, 30,60 and $120 \mathrm{pg} / \mathrm{ml}$. In addition to the blank wells, $100 \mu \mathrm{l}$ horseradish peroxidase (HRP)-labeled detection antibody (cat. no. ab181658, 1:1,000, Abcam, Cambridge, USA) was added to each well of the standard wells and sample wells. The wells were sealed with a sealing membrane and following incubation at $37^{\circ} \mathrm{C}$ for $60 \mathrm{~min}$, the liquid was removed, the plate was dried with absorbent paper and the plate was repeatedly washed with PBS 5 times. Then, $50 \mu 1$ of each of the substrates $\mathrm{A}$ and $\mathrm{B}$ were added to each well, and the mixture was incubated at $37^{\circ} \mathrm{C}$ for $15 \mathrm{~min}$ in the dark. Finally, $50 \mu \mathrm{l}$ of the stop solution was added to each well, the OD value of each well was measured by a microplate reader at a wavelength of $450 \mathrm{~nm}$ within $15 \mathrm{~min}$ and the protein concentration calculated according to the standard curve.

$R N A$ extraction and reverse transcription-quantitative [(RT- $q)$ $P C R]$. Spinal cord tissues and $10^{6}$ microglia of rats in each group were homogenized with Polytron PT100 (Kinematica AG) and $1 \mathrm{ml}$ RNAiso Plus (TaKaRa, Tokyo, Japan) was added 
to extract total RNAs and then purified by using GeneJET RNA Purification Kit (Thermo Fisher Scientific, Shanghai, China) according to the manufacturer's protocols. RT-qPCR was performed by using PrimeScript RT reagent kit (TaKaRa, Tokyo, Japan) according to the manufacturer's protocol. $\mathrm{RT}$ reaction was conducted for $15 \mathrm{~min}$ at $42^{\circ} \mathrm{C}$ followed by $5 \mathrm{~min}$ at $98^{\circ} \mathrm{C}$ and the reaction volume was $20 \mu \mathrm{l}$. The qPCR thermocycling conditions were: $95^{\circ} \mathrm{C}$ for $30 \mathrm{sec}$ followed by 40 cycles at $95^{\circ} \mathrm{C}$ for $5 \mathrm{sec}$ and $60^{\circ} \mathrm{C}$ for $30 \mathrm{sec}$ and the reaction volume was $25 \mu \mathrm{l}$. PCR primers were synthesized by Gene Pharma (GenePharma, Co., Ltd, Shanghai, China) and sequences are listed in Table I. The levels of mRNA expression were detected by SYBR Premix Ex Taq II Takara Bio, Inc.). The mRNA expressions of MALAT1 and AQP9 were normalized to GAPDH and miR-154-5p was normalized to $\mathrm{U} 6$ and $2^{-\Delta \Delta \mathrm{Cq}}$ method was used to calculate the relative gene expressions (28).

Protein extraction and western blot analysis. Total protein was extracted from spinal cord tissue and microglia of each group by using a RIPA lysis buffer (Beyotime Institute of Biotechnology) and the protein concentration was measured using a BCA Protein Assay kit (Thermo Fisher Scientific, Inc.). Proteins $(50 \mu \mathrm{g})$ were added to $10 \%$ SDS-PAGE and then transferred onto PVDF membranes (GE Healthcare Life Sciences), which were blocked with $5 \%$ non-fat milk in Tris-buffered saline at $25^{\circ} \mathrm{C}$ for $60 \mathrm{~min}$. Then the membranes were incubated with primary antibodies overnight at $4^{\circ} \mathrm{C}$. AQP9 $(1: 1,000$, cat. no. ab15129, $35 \mathrm{kDa}$, Abcam) and GAPDH (1:10,000, cat. no. ab181602, $36 \mathrm{kDa}$, Abcam). Following washing with Tris Buffered saline Tween (20\% Tween) TBST for 4 times, the membrane was then incubated with the secondary antibody, goat anti-Rat IgG (1:10,000, cat. no. ab150165, Abcam), for $1 \mathrm{~h}$ at room temperature. Protein bands were detected by Pierce ECL western blot substrate (Thermo Fisher Scientific, Inc.) with ECL detection system (Thermo Fisher Scientific, Inc.). Quantity One software (version 4.6.2, Bio-Rad Laboratories, Inc.) was used to quantify relative protein expression.

Pain threshold assessment. Pain behavior indicators were detected following lentivirus infection. The paw withdrawal threshold (PWT/g) and the paw withdrawal latency (PWL/s) of the sham group, CCI-LV-NC and CCI-LV-shMALAT1 groups were respectively detected at $0,1,3,7$ and 14 days. PWT was used to evaluate the mechanical allodynia. Rats of each group were placed in a transparent plastic box with metal mesh padding. The hind paw of the rats was placed in a calibrated Von Frey fiber and stimulation was given to them, then the minimum fiber strength was recorded. PWL was used to evaluate the thermal hyperalgesia and rats in each group were housed in a thick plexiglass box and tested by thermal radiation stimulation. The initial threshold of the base leg was set to $10 \mathrm{sec}$, and the time between the start of stimulation and the cessation of the action of the paws was recorded (PWL/s).

Bioinformatics analysis. Target genes were predicted through different bioinformatics databases, including starBase V2.0 (29) and TargetScan 7.1 (http://www.targetscan. org/vert_71/). First, the miRNAs that may bind to MALAT1 were analyzed and the miRNA in the two databases was taken
Table I. RT-qPCR primer sequences

\begin{tabular}{ll}
\hline Gene names & \multicolumn{1}{c}{ Primer sequences } \\
\hline MALAT1 & Forward: 5'-AAAGCAAGGTCTCCCCACAA \\
& G-3' \\
& Reverse: 5'- GGTCTGTGCTAGATCAAAAG \\
& GCA-3' \\
miR-154-5p & Forward: 5'-CGCGAATTCGCATCTAGGAC \\
& CTCCATCAC -3' \\
& Reverse: 5'-ACGGGATCCGAACCATCCCT \\
& TCACTTACC -3' \\
AQP9 & Forward: 5'-TCTCGTCCTTGGATGTGGC-3' \\
& Reverse: 5'-CAAAAGACACCGCTGGGTT \\
& G-3' \\
GAPDH & Forward: 5'-GGAGTCCACTGGTGTCTTC \\
& A-3' \\
& Reverse: 5'-GGGAACTGAGCAATTGGTG \\
& G-3' \\
& Forward: 5'-CTCGCTTCGGCAGCACA-3' \\
& Reverse: 5'-AACGCTTCACGAATTTGC \\
U6 & GT-3' \\
\hline
\end{tabular}

MALAT1, metastasis associated lung adenocarcinoma transcript; miR, microRNA; AQP, aquaporin

for further verification; miR-154-5p was identified. Second, the potential target genes of miR-154-5p were predicted with the two bioinformatics databases; 104 target genes had been reported, including transcription factors, kinases, channel proteins and cancer-related genes. The top 5 genes were E2F5, HDHD2, SOS2, AQP9 and HNRNPR. The most likely target genes were selected for experimental validation.

Luciferase assays. The MALAT/AQP9 wild type (WT) sequences or mutant (mut) sequences in 3'-UTR containing the miR-154-5p binding site were constructed and subcloned into the pGL3 basic plasmid (Addgene, Inc.). 293 cells were seeded in 48-well plates for $24 \mathrm{~h}$. Then miR-154-5p mimics and miR-NC were co-transfected into 293 cells with pGL3-WT/mut-MALAT1 for $24 \mathrm{~h}$. Plasmids $(200 \mathrm{ng})$ were mixed with Lipofectamine ${ }^{\circledR} 2000$ (Invitrogen; Thermo Fisher Scientific, Inc.) and DMEM medium for 20 mins, then the mixtures were added into 293 cells for $24 \mathrm{~h}$. Following transfection for $24 \mathrm{~h}$, the cells were lysed and the activity of firefly luciferase and Renilla luciferase was measured by using dual-luciferase reporter assay (Promega Corporation). The ratio of the two identified the relative activity of luciferase.

Statistical analysis. All the data were expressed as the mean \pm standard deviation, the significance between groups was analyzed by Student's t-test and multiple comparison between the groups was performed by the SNK method following one-way ANOVA. All statistical analyses were performed using SPSS 19.0 (IBM Corp.) and GraphPad Prism 6.0 (GraphPad Software, Inc.). $\mathrm{P}<0.05$ was considered to indicate a statistically significant difference. 


\section{Results}

MALAT1 is increased in CCI rat models. To explore the roles of MALAT1 in neuropathic pain, the expressions of MALAT1 in spinal cord tissue and microglia of CCI rats were detected for the first time. The expression of MALAT1 in spinal cord tissue and microglia of CCI rats was significantly increased with time, compared with the sham group (Fig. 1A and B; $\mathrm{P}<0.05)$, which indicated that MALAT1 was increased in CCI rat models.

Inhibition of MALAT1 reduces the incidence of neuropathic pain. To further investigate of the function of MALAT1 in CCI model rats, recombinant lentiviral LV-shMALAT1 and LV-NC were injected into CCI rats. After 14 days, spinal cord tissue and microglia were isolated from rats and MALAT1 levels were detected by RT-qPCR. The results demonstrated that the expression of MALAT1 was significantly increased in CCI LV-NC group, while it was repressed following LV-shMALAT1 transfection in spinal cord tissue and microglia (Fig. 2A and B; $\mathrm{P}<0.05$ ). The neuropathic pain in each group of rats was evaluated using PWT and PWL. The results demonstrated that the PWT and PWL of CCI rats were significantly decreased with time dependence, compared with the sham group, while they were increased following LV-shMALAT1 transfection (Fig. $2 \mathrm{C}$ and $\mathrm{D} ; \mathrm{P}<0.05$ ). These results indicated that downregulation of MALAT1 in the CCI model promoted neuropathic pain in rats, whereas inhibition of MALAT1 reduced the incidence of neuropathic pain.

Inhibition of MALAT1 reduces the levels of inflammatory cytokines. To further explore whether MALAT1 served some roles in inflammation of CCI model rats, the expression levels of inflammatory cytokines in spinal cord tissue and microglia were detected using ELISA in each group. The results demonstrated that the levels of the inflammatory cytokines COX2, TNF- $\alpha$ and IL- 6 were significantly increased in the CCI model group, compared with the sham group, while these inflammatory factors were significantly decreased in MALAT1 inhibition model in both spinal cord tissue and microglia (Fig. 3A-F; P<0.05). These results indicated that inhibition of MALAT1 reduced the levels of inflammatory cytokines and mitigated inflammatory responses in CCI rats.

MALAT1 can directly bind with miR-154-5p. To investigate the molecular mechanism whereby MALAT1 promoted neuropathic pain in CCI rats, miRNAs were predicted by starBase v2.0 database and it was identified that miR-154-5p may be a target of MALAT1; therefore, the levels of miR-154-5p were detected by RT-qPCR. The results demonstrated that MALAT1 was decreased in LV-shMALAT1 group, while miR-154-5p was respectively increased, which might suggest that MALAT1 was negatively correlated with miR-154-5p (Fig. 4A and B; $\mathrm{P}<0.05$ ). The potential binding site with miR-154-5p was predicted by starBase v2 .0 database and sequences of wt-MALAT1 and mut-MALAT1 were constructed into luciferase reporter vector (Fig. 4C). The results demonstrated that the luciferase activity in cells co-transfected with miR-154-5p mimic and wt-MALAT1 was significantly decreased, while it was increased in mut-MALAT1 group in
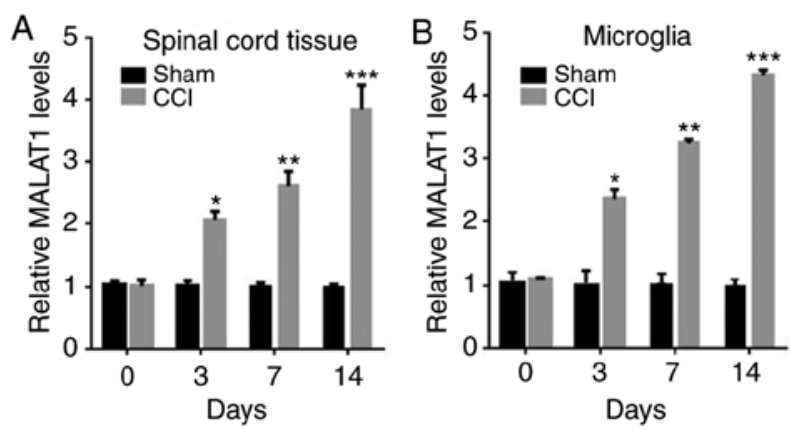

Figure 1. MALAT1 was increased in CCI rat models. The mRNA levels of MALAT1 in (A) spinal cord tissue and (B) microglia of rats were detected by RT-qPCR. Data are shown as mean \pm standard deviation based on at least three independent experiments, ${ }^{*} \mathrm{P}<0.05 ;{ }^{* *} \mathrm{P}<0.01 ;{ }^{* * *} \mathrm{P}<0.001$ vs. sham group. MALAT, metastasis associated lung adenocarcinoma transcript; CCI, chronic constriction injury; RT-qPCR, reverse transcription-quantitative PCR.

293 cells (Fig. 4D; P<0.05). In addition, LV-miR-154-5p was injected into CCI rats and PWT and PWL detected. The results showed that miR-154-5p was significantly increased following LV-miR-154-5p infection, which suggested that it was successfully infected into the rats and it was found that overexpression of miR-154-5p could have a similar effect to the inhibition of MALAT1 (Fig. 4E and F; P<0.05). These results indicated that MALAT1 could directly bind with miR-154-5p.

miR-154-5p overexpression inhibits inflammatory responses in CCI rats. MALAT1 could directly bind with miR-154-5p; however, the roles of miR-154-5p in CCI rats remained to be elucidated, so the expression of miR-154-5p was detected in CCI rats. The results demonstrated that the miR-154-5p of CCI rats was decreased in spinal cord tissue and microglia with time, compared with the sham group (Fig. 5A and B; $\mathrm{P}<0.05$ ). To further explore the roles of miR-154-5p in CCI rats, lentiviral vector LV-miR-154-5p was constructed and injected into the rats. After 14 days, spinal cord tissue and microglia were separated and the levels of miR-154-5p, COX2, TNF- $\alpha$ and IL-6 were detected by ELISA kits. The results demonstrated that miR-154-5p was significantly increased in the LV-miR-154-5p group, which indicated that LV-miR-154-5p was successfully transfected into CCI rats (Fig. 5C and D; $\mathrm{P}<0.05$ ). The levels of COX2, TNF- $\alpha$ and IL- 6 were significantly decreased in the LV-miR-154-5p group (Fig. 5E and $F$; $P<0.05$ ). These results suggested that miR-154-5p overexpression inhibited inflammatory responses in CCI rats.

miR-154-5p can directly bind with AQP9. To further explore the detailed mechanisms of miR-154-5p in the inhibition of the inflammatory responses in CCI rats, target genes were analyzed by the TargetScan database and AQP9, a member of the aquaporins, was predicted to be the target gene of miR-154-5p. Therefore, the expression of AQP9 was detected in CCI rats. The results demonstrated that mRNA and protein levels of AQP9 were significantly increased, while miR-154-5p was decreased in CCI rats in spinal cord tissue and microglia with time, compared with the sham group (Fig. 6A-F; P<0.05). To further explore the binding site, the potential binding site was predicted. A wt-AQP9 luciferase reporter vector (wt-AQP9) and a mut-AQP9 3'UTR luciferase reporter vector (mut-AQP9) 
A
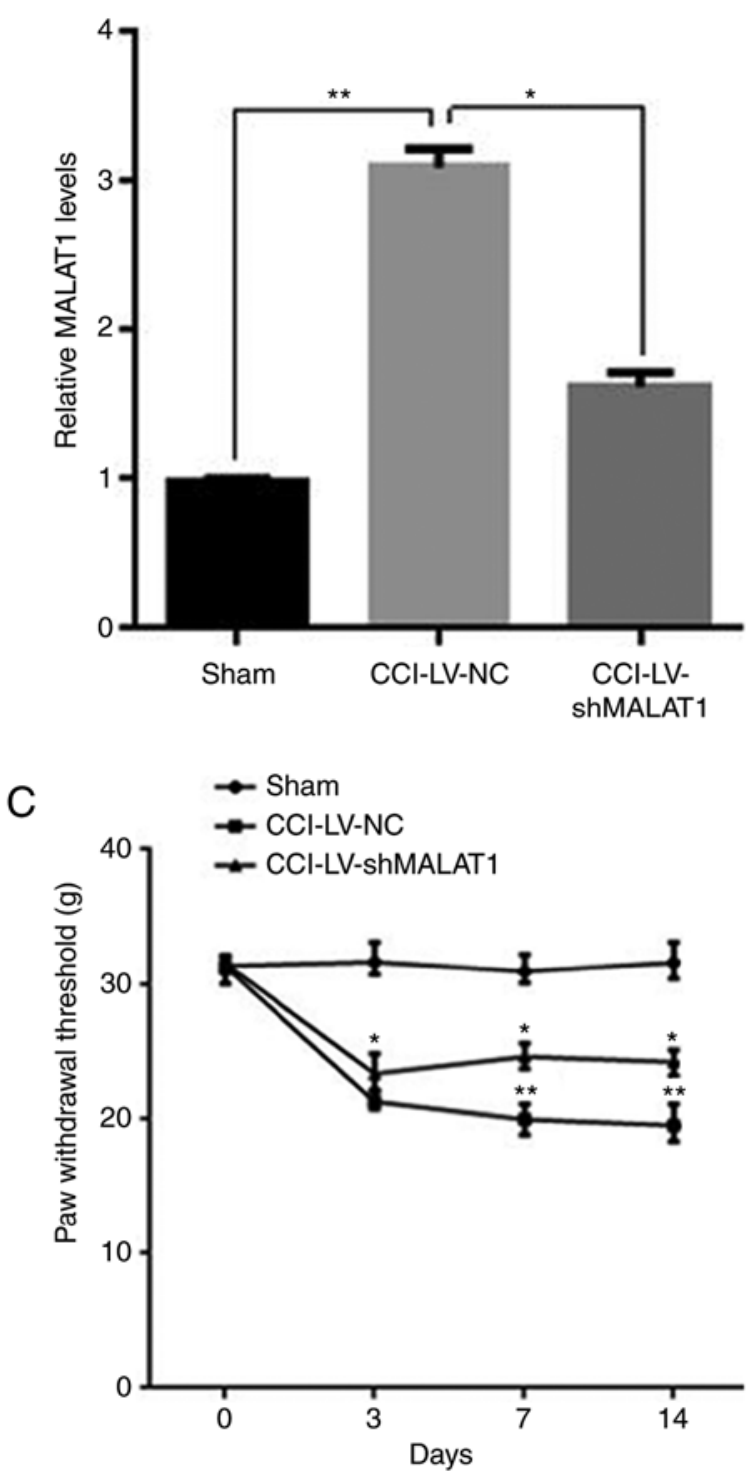

B
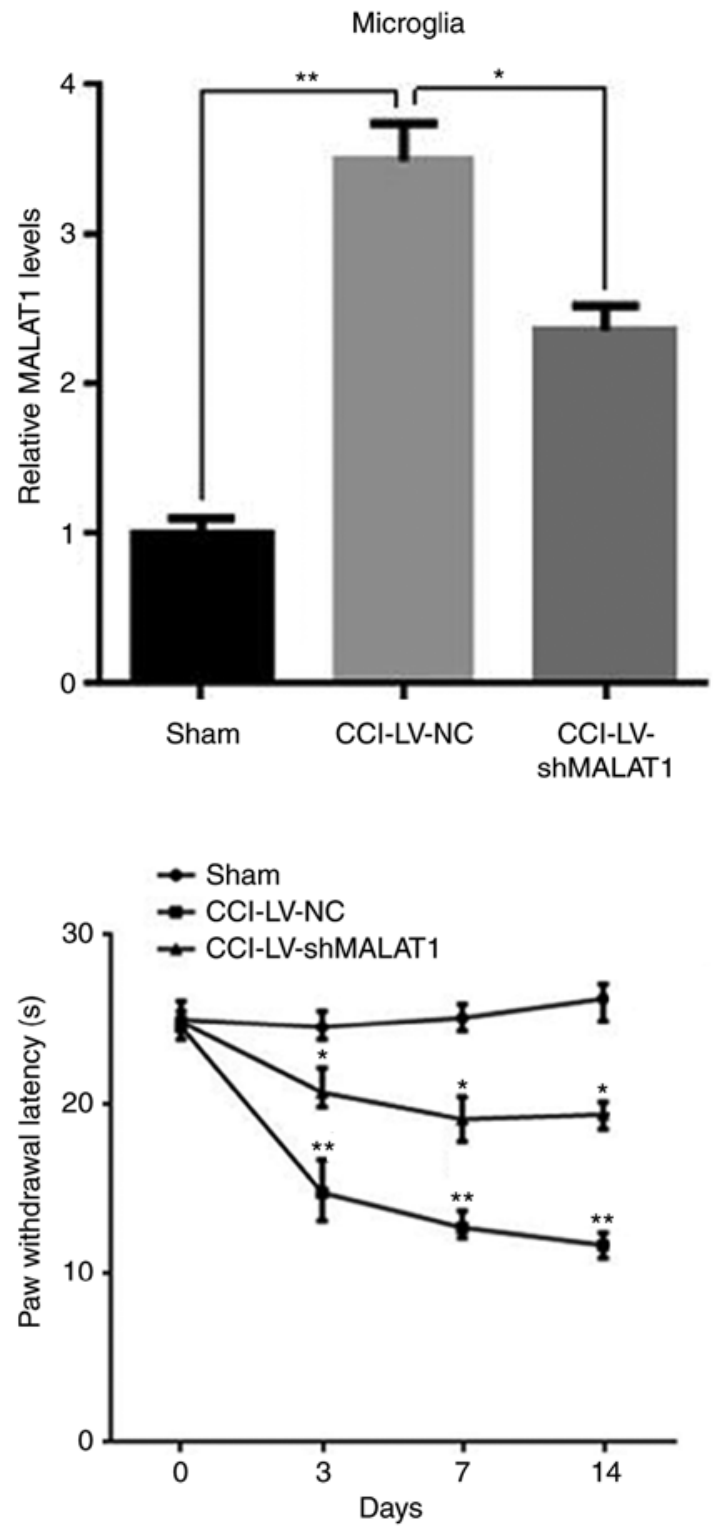

Figure 2. Inhibition of MALAT1 reduced the incidence of neuropathic pain. The mRNA levels of MALAT1 in (A) spinal cord tissue and (B) microglia of CCI rats were detected by RT-qPCR after LV-shMALAT1 transfection. The mechanical allodynia of (C) PWT and (D) thermal hyperalgesia of PWL were detected. Data are shown as mean \pm standard deviation based on at least three independent experiments. ${ }^{*} \mathrm{P}<0.05$ vs. LV-NC CCI group, ${ }^{* * *} \mathrm{P}<0.01$ vs. sham group. MALAT, metastasis associated lung adenocarcinoma transcript; CCI, chronic constriction injury; RT-qPCR, reverse transcription-quantitative PCR; PWT, paw withdrawal threshold; PWL, paw withdrawal latency; LV, lentivirus; NC, negative control; sh, short hairpin.

were constructed (Fig. 6G). Compared with control group, the luciferase activity in co-transfection with miR-154-5p mimic and wt-AQP9 was significantly decreased, while it was increased in mut-AQP9 group in 293 cells (Fig. 6H; $\mathrm{P}<0.05$ ). These data indicated that miR-154-5p could directly bind with AQP9.

MALAT1 promotes neuropathic pain via the miR-154-5p/AQP9 axis in CCI rats. From the above results, it was hypothesized that MALAT1 might promote neuropathic pain through miR-154-5p/AQP9 axis in CCI rats. To confirm this hypothesis, recombinant lentiviral vector LV-shAQP9 was constructed and injected into CCI rats. AQP9 was detected by RT-qPCR and western blotting. The results demonstrated that the expression of AQP9 was significantly increased in spinal cord tissue and microglia of CCI rats compared with the sham group, while it was significantly decreased in LV-shAQP9 (Fig. 7A-F; P<0.05). The mechanical pain threshold in each group of rats was evaluated using PWT and PWL. The results demonstrated that the PWT and PWL of CCI rats were significantly decreased with time, compared with the sham group, but significantly increased in LV-shAQP9 group (Fig. 7G and H; $\mathrm{P}<0.05$ ), which suggested that AQP9 could reduce the neuropathic pain. To further explore whether MALAT1 interacted with AQP9 through miR-154-5p, miR-154-5p inhibitor was added into LV-shMALAT1 rats and the expressions of miR-154-5p and AQP9 were detected by RT-qPCR and western blotting. The results demonstrated that miR-154-5p was significantly upregulated following infection with shMALAT1, while it was decreased in spinal cord tissue following the addition of miR-154-5p inhibitor (Fig. 7I; P<0.05). AQP9 expression was significantly decreased following infecting with 

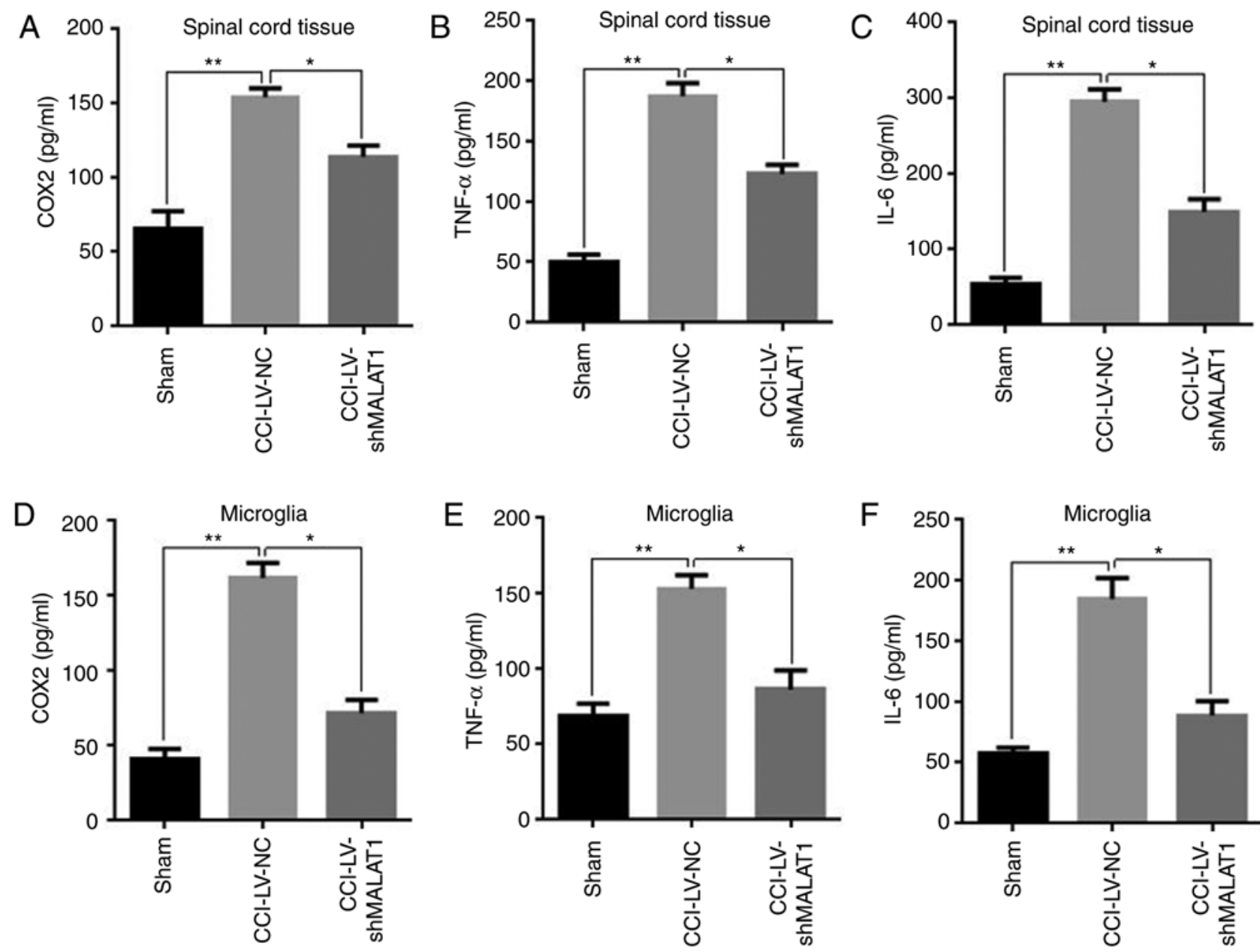

Figure 3. Inhibition of MALAT1 reduced the levels of inflammatory cytokines. (A-F) Inflammatory cytokines in spinal cord tissue and microglia were detected by ELISA. Data are shown as mean \pm standard deviation based on at least three independent experiments, ${ }^{*} \mathrm{P}<0.05$ vs. LV-NC CCI group, ${ }^{* *} \mathrm{P}<0.01$ vs. sham group. MALAT, metastasis associated lung adenocarcinoma transcript; COX, cyclooxygenase; TNF, tumor necrosis factor; IL, interleukin; LV, lentivirus; $\mathrm{NC}$, negative control; sh, short hairpin.

shMALAT1, while it was significantly increased following the addition of miR-154-5p inhibitor (Fig. 7J and K; P<0.05) and the similar results had been found in microglia (Fig. 7L-N; $\mathrm{P}<0.05)$. These results indicated that MALAT1 promoted the occurrence of neuropathic pain in CCI rats by targeting the miR-154-5p/AQP9 axis.

\section{Discussion}

Neuropathic pain is a problem following spinal surgery for patients and may be associated with abnormal sensations called dysesthesia, or pain from normally non-painful stimuli, called allodynia (1-3,30,31). Although progress has been made in clinical treatment, a number of patients still suffer chronic pain and psychological problems (4). However, the molecular mechanism of neuropathic pain remains to be elucidated.

In recent years, IncRNAs and miRNAs have attracted much attentions due to their potential in the diagnosis and treatment of neuropathic pain. Therefore, it is worth studying the specific mechanism of non-coding RNAs in neuropathic pain. MALAT1 is widely considered to be involved in human cancer, vascular disease and nervous system diseases (1-3,30,31). However, the specific function of MALAT1 in neuropathic pain CCI model rats is still unclear. The present study identified that MALAT1 expression was upregulated in bone marrow tissue and microglia in CCI rats, compared with the sham group. MALAT1 may serve a role in a CCI model and in neuropathic pain.

To further investigate the function of MALAT1 in neural system of CCI model rats, LV-shMALAT1 lentivirus was administered by intrathecal injection and microglia were isolated from the bone marrow tissue of each group. The results demonstrated that upregulation of MALAT1 in the CCI model promoted neuropathic pain in rats, whereas inhibition of MALAT1 reduced the incidence of neuropathic pain. The levels of inflammatory cytokines COX2, TNF- $\alpha$, and IL-6 were significantly increased in the CCI model group, while these inflammatory factors were significantly decreased in the MALAT1 inhibition model. These results indicated that inhibition of MALAT1 reduced the levels of inflammatory cytokines and mitigated inflammatory responses in CCI rats.

To explore the molecular mechanism by which MALAT1 regulated the neuropathic pain in rats and using starBase v2.0, it was hypothesized that there was a potential binding site between MALAT1 and miR-154-5p. Then the levels of 

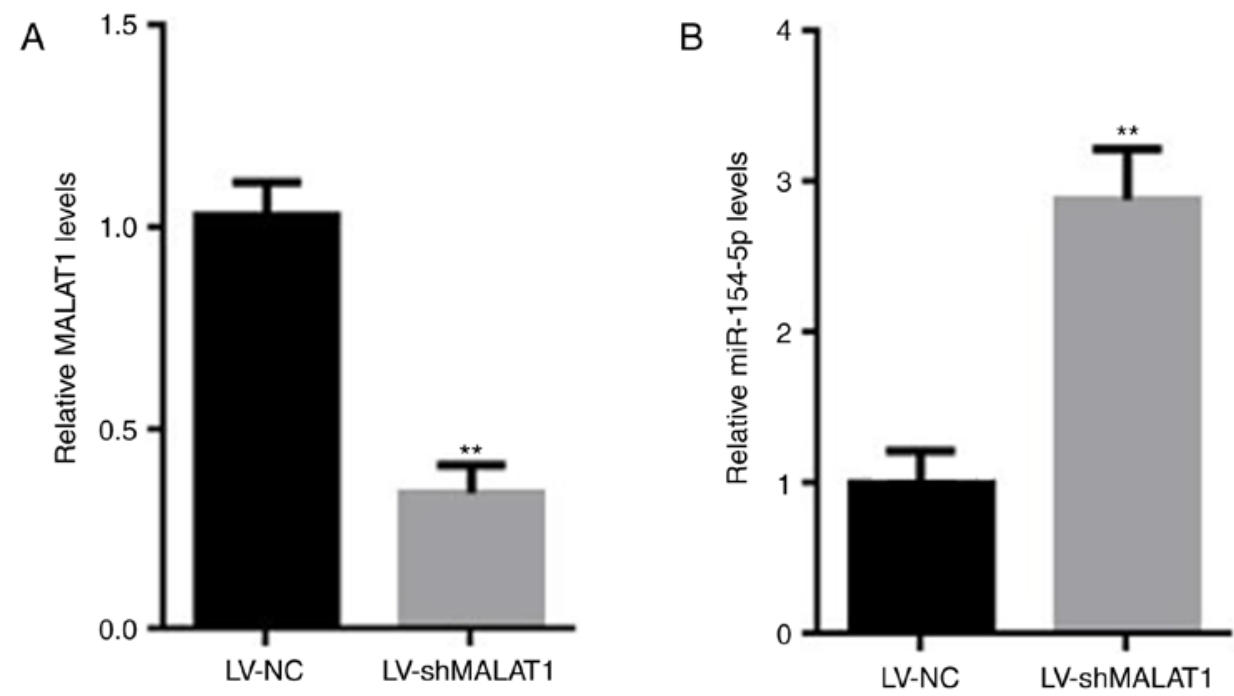

$\begin{array}{rr}\text { C MALAT1 WT-3'UTR } & \text { 5'-ataaggaataaATAACCTc-3' } \\ \text { miR-154-5p } & \text { 3'-ttccgttgtgccTATTGGAt-5' } \\ \text { MALAT1 mut-3'UTR } & \text { 5'-ataaggaataaTATTGGAC-3' }\end{array}$

$\mathrm{D}$

$293 \mathrm{~T}$
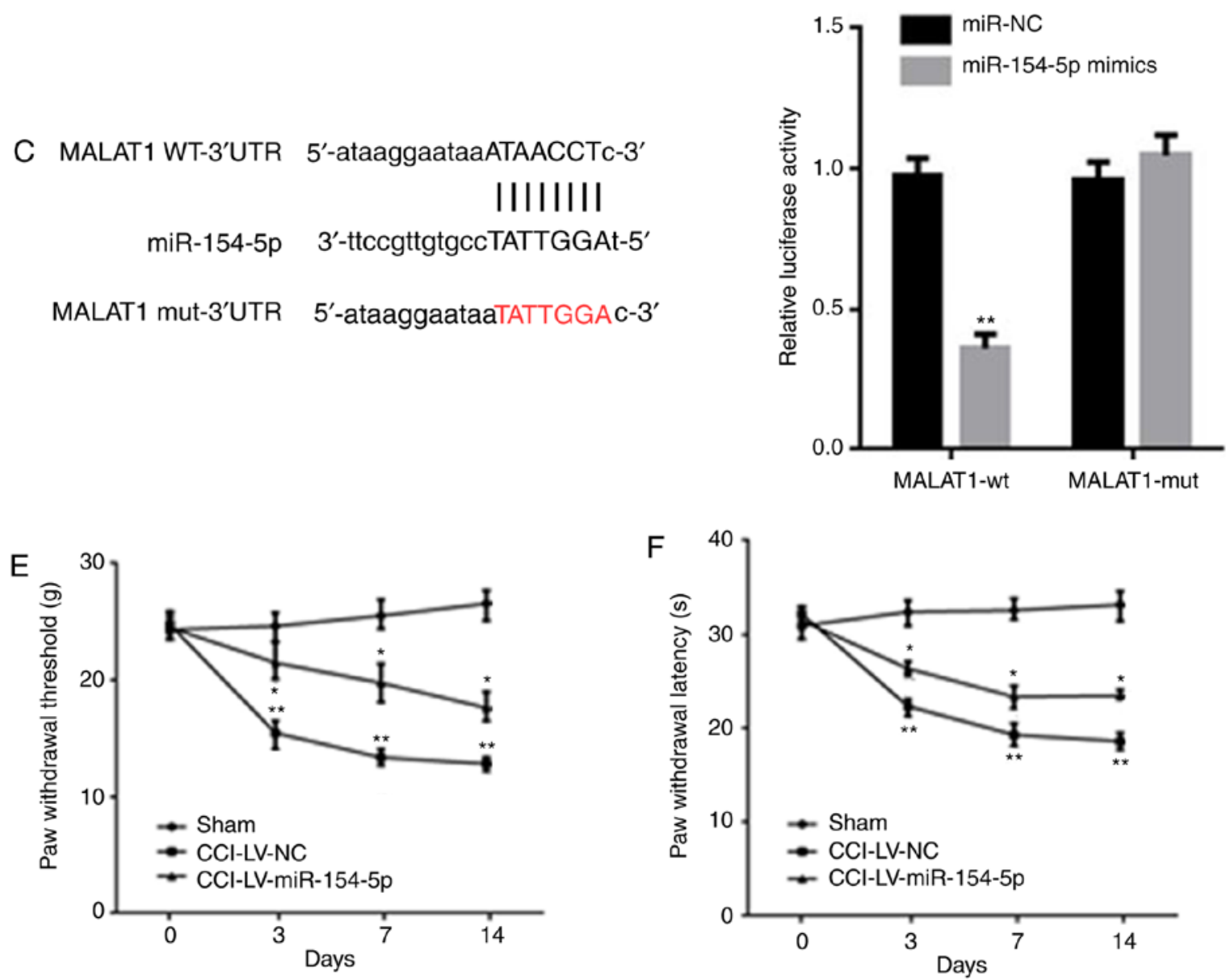

Figure 4. MALAT1 could directly bind with miR-154-5p. (A, B) The mRNA levels of (A) MALAT1 and (B) miR-154-5p in microglia of CCI rats were detected by RT-qPCR. (C) Potential binding sites between MALAT1 and miR-154-5p was predicted by starBase v2.0 database. (D) The luciferase reporter assay was performed to determine the binding site. (E) PWT and (F) PWL were detected after LV-miR-154-5p injection into CCI rats. Data are shown as mean \pm standard deviation based on at least three independent experiments. ${ }^{*} \mathrm{P}<0.05,{ }^{* *} \mathrm{P}<0.01$ vs. LV-NC CCI group. MALAT, metastasis associated lung adenocarcinoma transcript; miR, microRNA; CCI, chronic constriction injury; RT-qPCR, reverse transcription-quantitative PCR; PWT, paw withdrawal threshold; PWL, paw withdrawal latency; LV, lentivirus; NC, negative control; sh, short hairpin; wt, wild type; mut, mutation.

miR-154-5p and MALAT1 were detected by RT-qPCR. The results demonstrated that MALAT1 was decreased in the LV-shMALAT1 group, while miR-154-5p was respectively increased, which may suggest that MALAT1 was negatively correlated with miR-154-5p. To further explore whether MALAT1 bound with miR-154-5p, the wt-MALAT1 luciferase reporter vector and a mut-MALAT1 3'UTR luciferase reporter vector were constructed and the luciferase reporter 
A

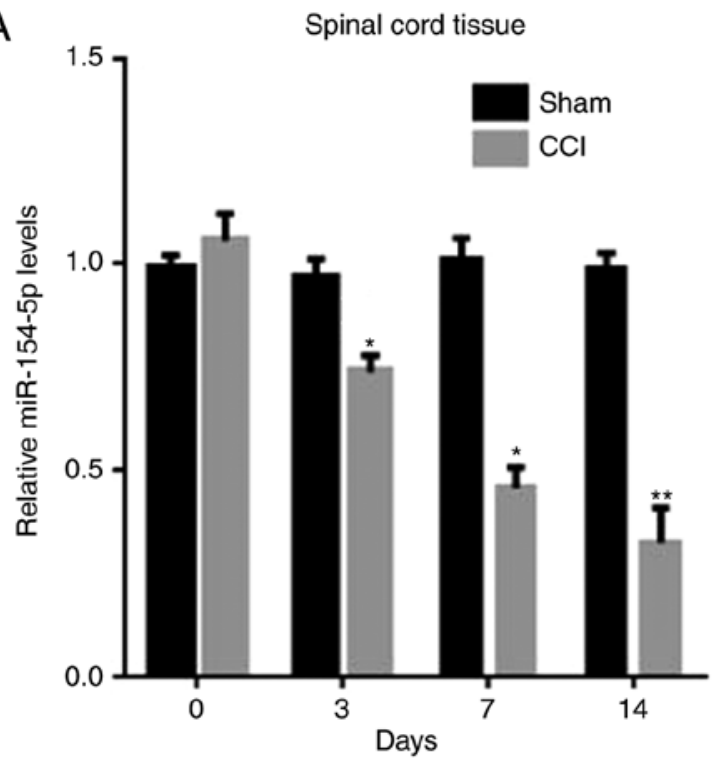

C

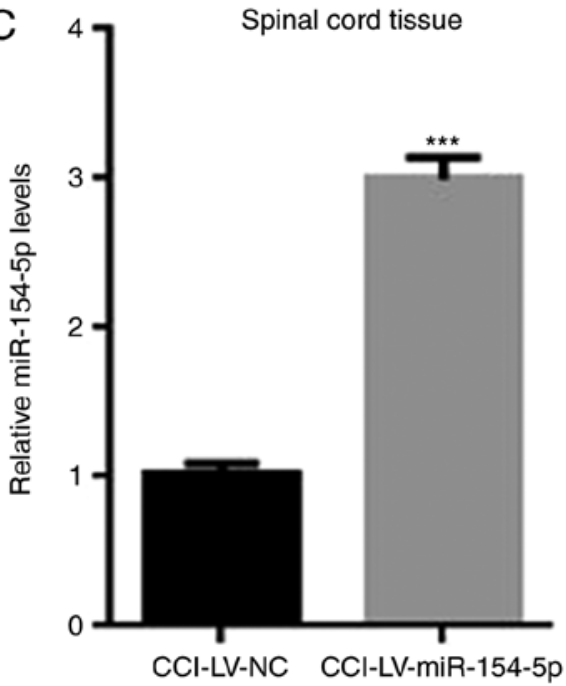

E

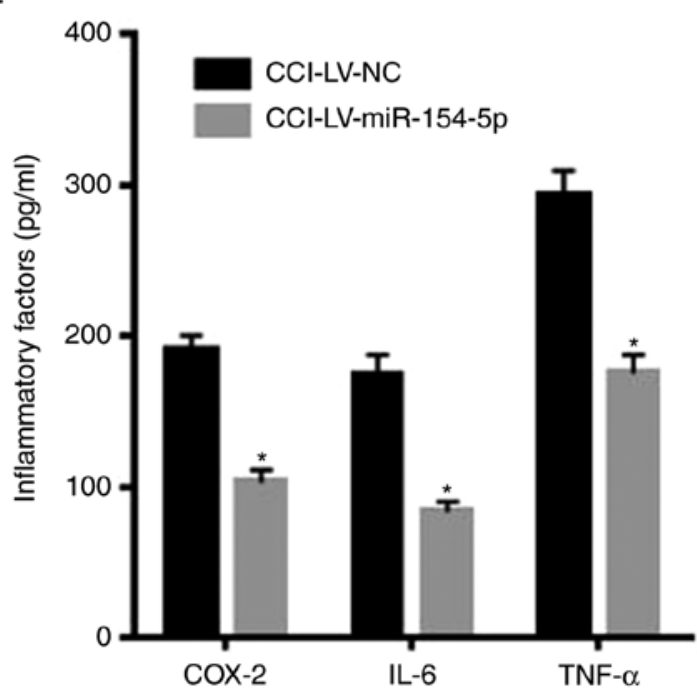

B
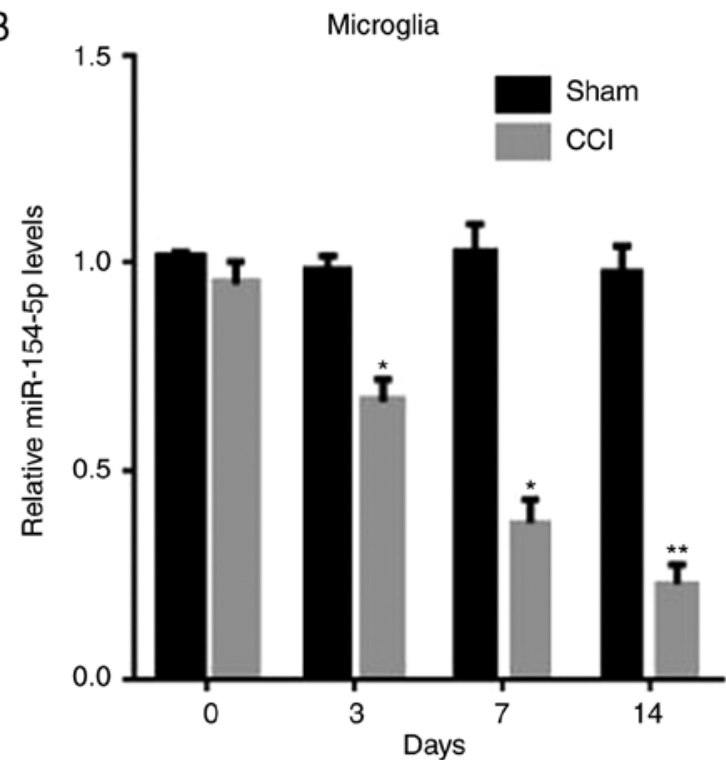

D

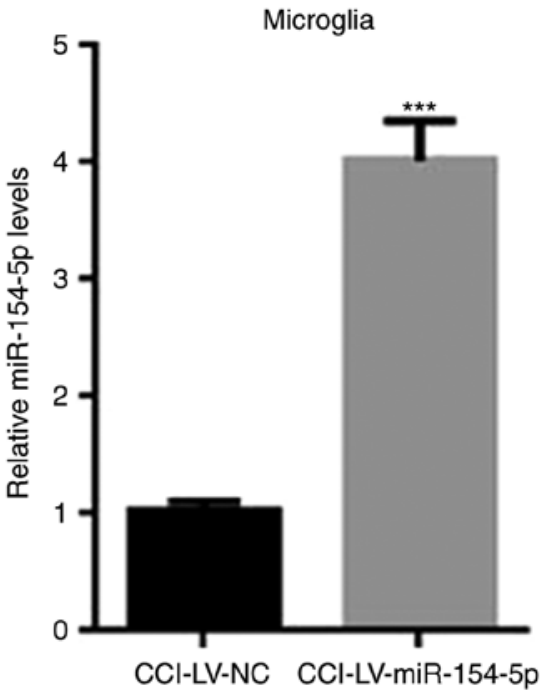

Microglia

F

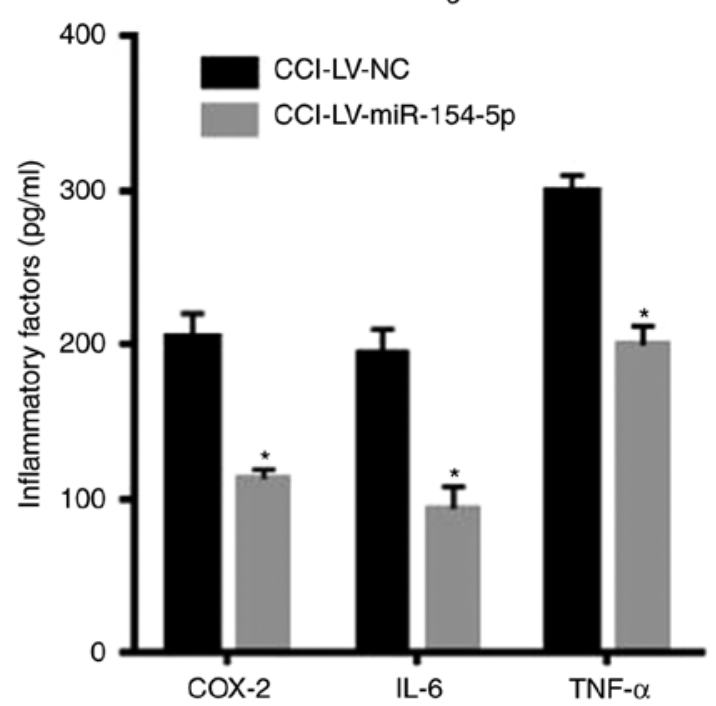

Figure 5. miR-154-5p overexpression inhibited inflammatory responses in CCI rats. The mRNA levels of miR-154-5p in (A) spinal cord tissue and (B) microglia of sham and CCI rats were detected by RT-qPCR. The mRNA levels of miR-154-5p in (C) spinal cord tissue and (D) microglia were detected after LV-miR-154-5p injection into CCI rats. Inflammatory cytokines in (E) spinal cord tissue and (F) microglia were detected by ELISA. Data are shown as mean \pm standard deviation based on at least three independent experiments, ${ }^{*} \mathrm{P}<0.05,{ }^{* *} \mathrm{P}<0.01,{ }^{* * *} \mathrm{P}<0.001$ vs. LV-NC CCI group. miR, microRNA; CCI, chro nic constriction injury; RT-qPCR, reverse transcription-quantitative PCR; LV, lentivirus; COX, cyclooxygenase; TNF, tumor necrosis factor; IL, interleukin; $\mathrm{NC}$, negative control. 
A

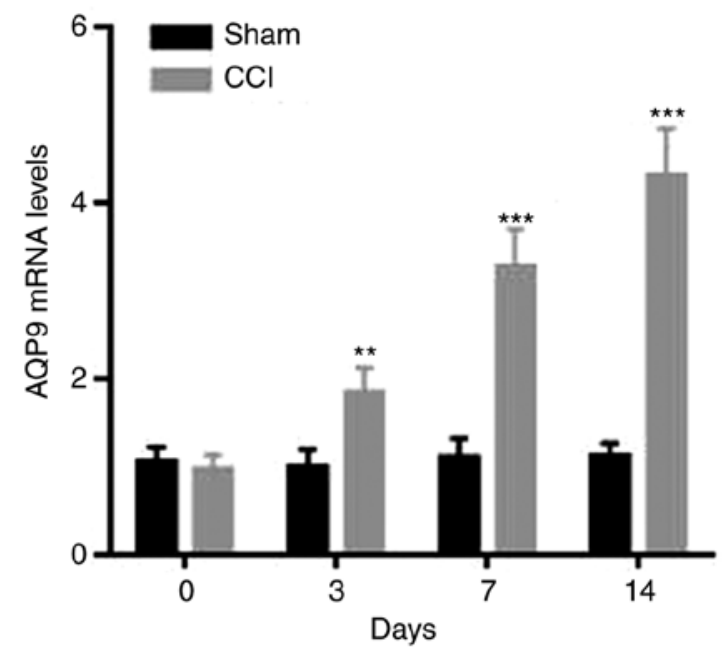

C

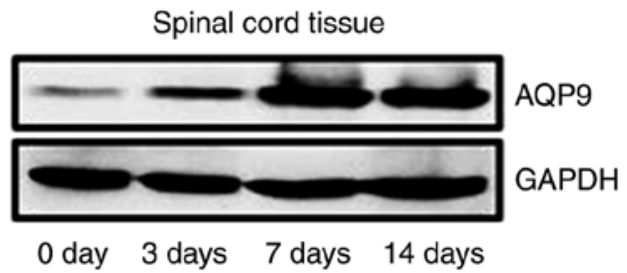

E

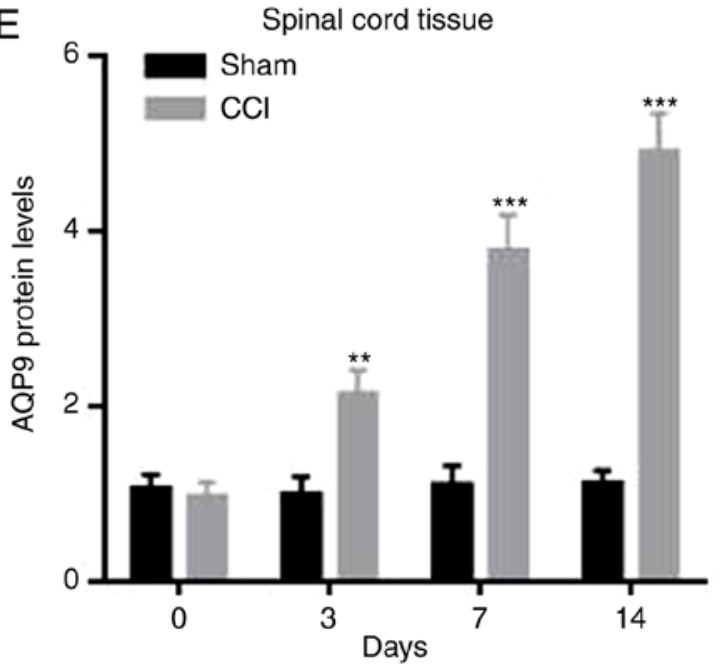

G AQP9 WT-3 UTR 5 -ucuuuaagucuauaAUAACCUA-3 |||||| $\mid$

miR-154-5p 3 -gcuuccguugugccUAUUGGAU-5

AQP9 mut-3 UTR 5 -ucuuuaagucuauaUAUUGGAU-3
B

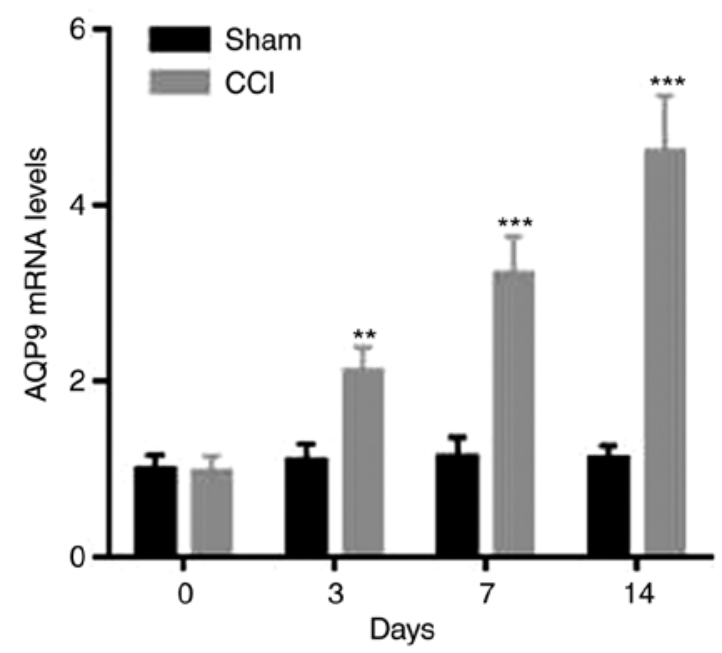

D

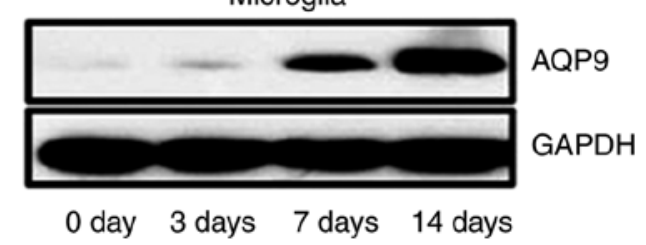

$\mathrm{F}$
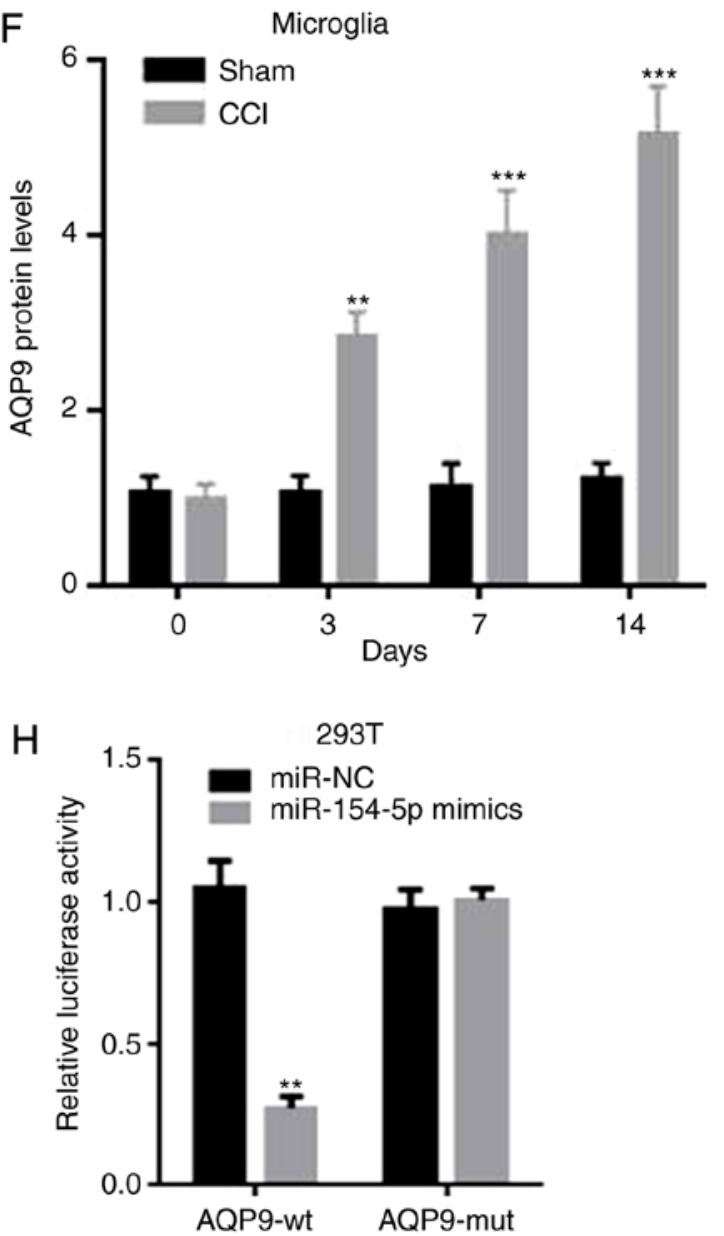

Figure 6. miR-154-5p could directly bind with AQP9. (A-F) mRNA levels of AQP9 in (A) spinal cord tissue and (B) microglia of CCI rats were detected using RT-qPCR. Protein levels of AQP9 were detected in (C) spinal cord tissue and (D) microglia of CCI rats using western blot analysis, with subsequent quantification of levels in (E) spinal cord tissue and (F) microglia. (G) Potential binding site between AQP9 and miR-154-5p was predicted by TargetScan database. $(\mathrm{H})$ The luciferase reporter assay was performed to determine the binding site. Data are shown as mean \pm standard deviation based on at least three independent experiments, ${ }^{* *} \mathrm{P}<0.01,{ }^{* * *} \mathrm{P}<0.001$ vs. sham group. miR, microRNA; AQP, aquaporin; $\mathrm{CCI}$, chronic constriction injury; RT-qPCR, reverse transcription-quantitative PCR; NC, negative control. 
A
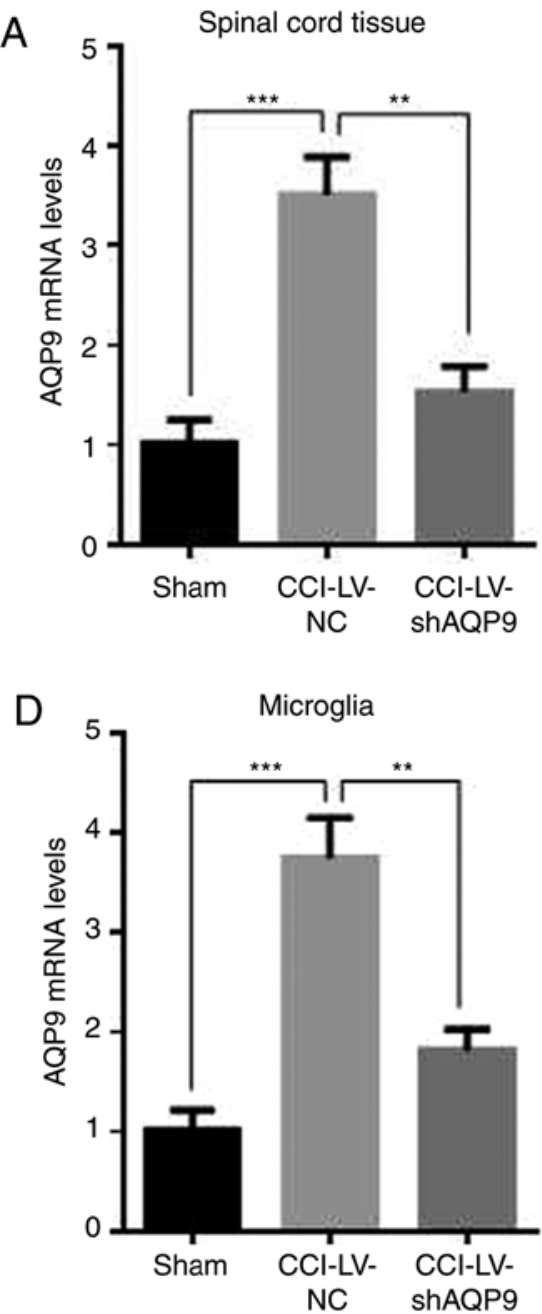

G

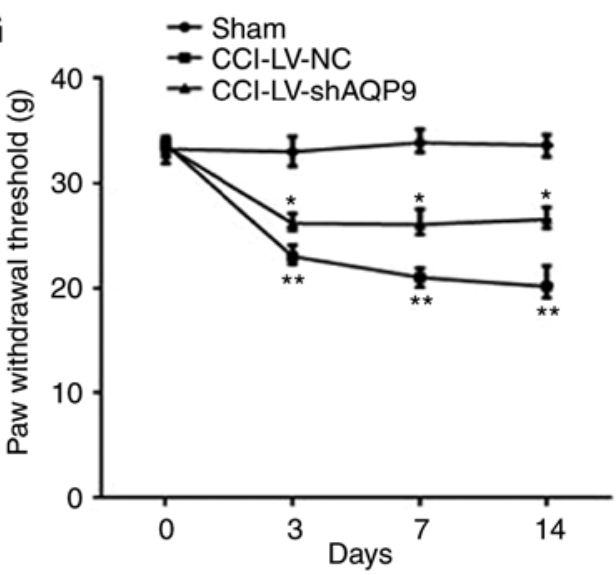

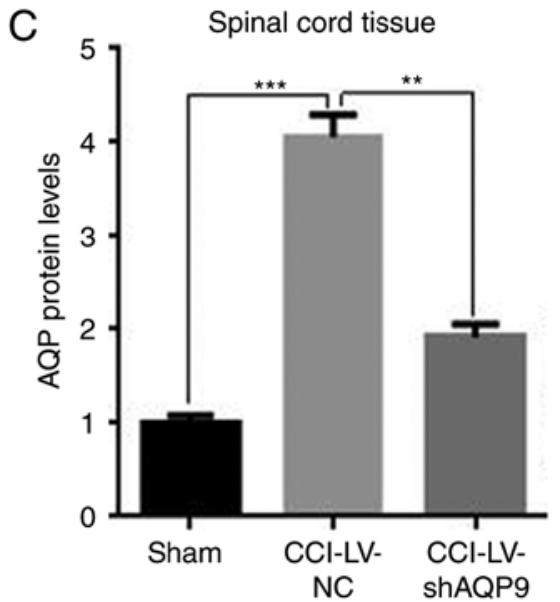

$\mathrm{F}$

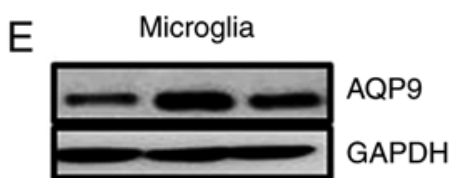

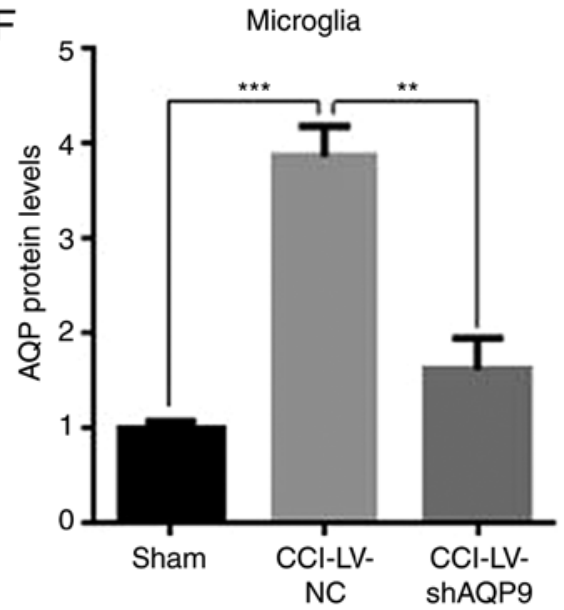

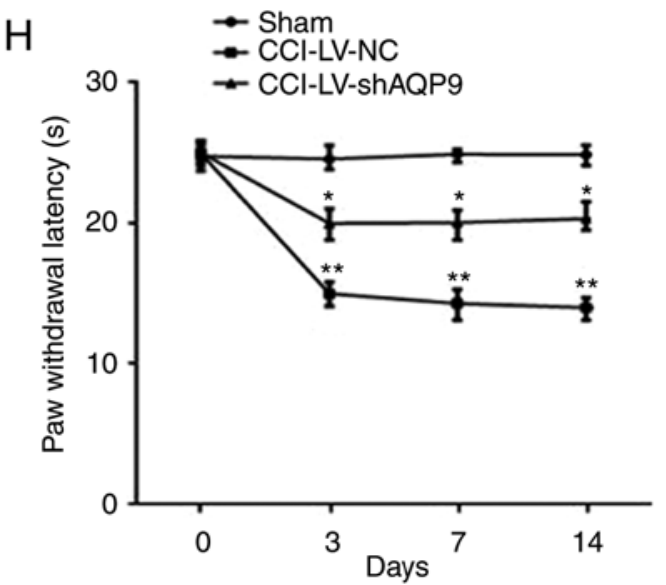

Figure 7. MALAT1 promoted neuropathic pain through miR-154-5p/AQP9 axis in CCI rats. mRNA levels of AQP9 in (A) spinal cord tissue. Protein levels of (B) spinal cord tissue with (C) subsequent quantification. (D) microglia detected after LV-shAQP9 transfection using RT-qPCR. Protein levels of (E) microglia with (F) subsequent quantification. (G) The mechanical allodynia of PWT and (H) thermal hyperalgesia of PWL were detected. Data are shown as mean \pm standard deviation based on at least three independent experiments. ${ }^{*} \mathrm{P}<0.05,{ }^{* *} \mathrm{P}<0.01$ vs. LV-NC CCI group; ${ }^{* * *} \mathrm{P}<0.001$ vs. sham group.

gene assay was performed. The results demonstrated that MALAT1 could directly bind with miR-154-5p. However, the roles of miR-154-5p in CCI rats remained unclear.

It has been identified that the dysregulation of miR-154-5p is common in a number of cancers, including colorectal cancer, hepatocellular carcinoma and prostate cancer $(18,32-34)$. Studies have reported that miRNA-154-5p can inhibit the proliferation, migration and invasion of prostate cancer cells by targeting E2F5 (18), and miRNA-154-5p inhibits cell proliferation and metastasis through targeting PIWIL1 (19). However, the role of miR-154-5p in neuropathic pain remains to be elucidated. Therefore, the expression of miR-154-5p was detected in CCI rats. The results demonstrated that miR-154-5p in CCI rats was decreased in spinal 

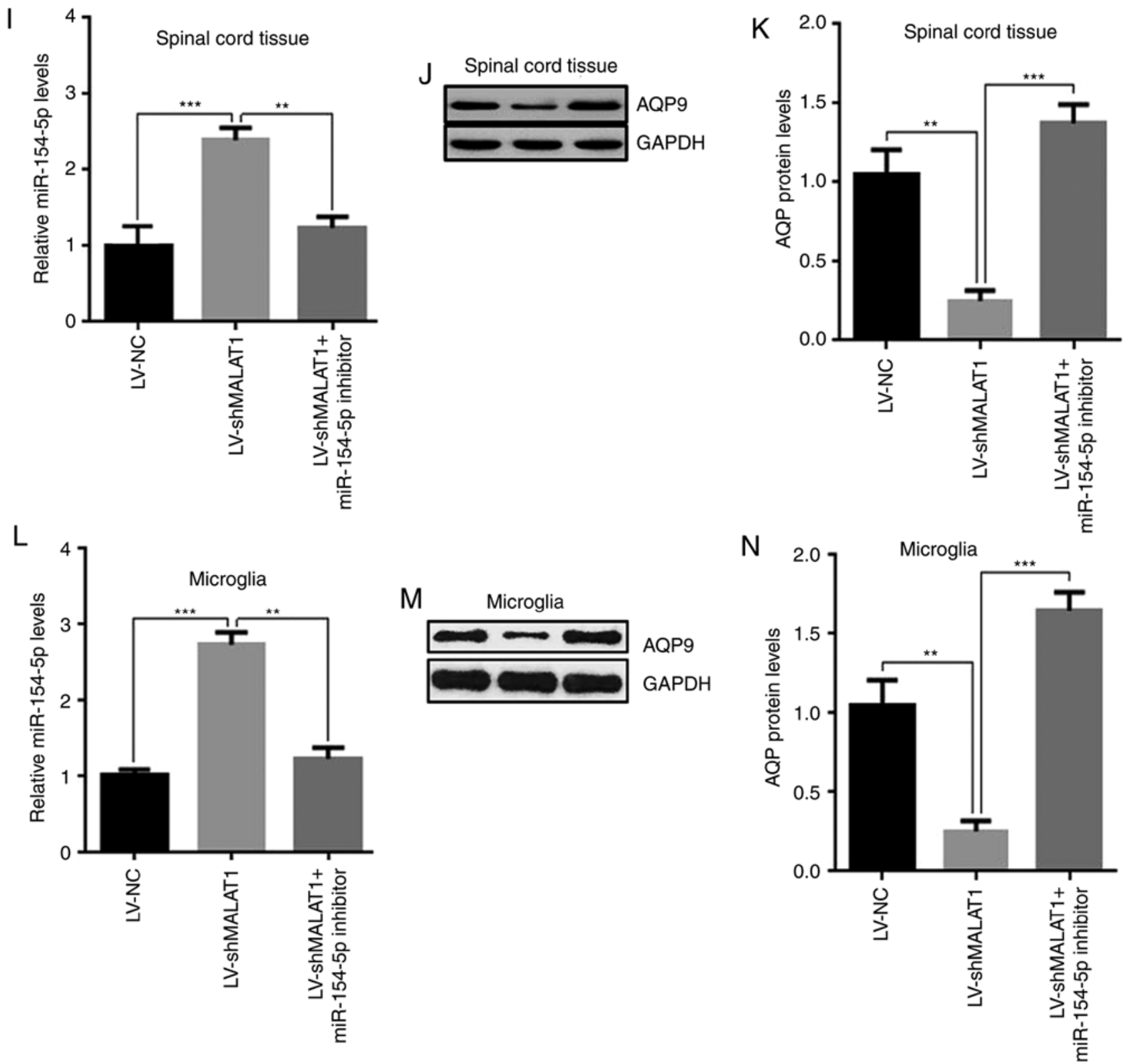

Figure 7. Continued. MALAT1 promoted neuropathic pain through miR-154-5p/AQP9 axis in CCI rats. The levels of miR-154-5p were detected after adding LV-shAMALAT1 with miR-154-5p inhibitor in (I) spinal cord tissue and (L) microglia. Protein levels of AQP9 were detected in (J) spinal cord tissue and (M) microglia of CCI rats using western blot analysis, with subsequent quantification of levels in $(\mathrm{K})$ spinal cord tissue and $(\mathrm{N})$ microglia. Data are shown as mean \pm standard deviation based on at least three independent experiments. ${ }^{* *} \mathrm{P}<0.01$ vs. LV-NC CCI group; ${ }^{* * *} \mathrm{P}<0.001$ vs. sham group. MALAT, metastasis associated lung adenocarcinoma transcript; miR, microRNA; AQP, aquaporin; CCI, chronic constriction injury; LV, lentivirus; sh, short hairpin; PWT, paw withdrawal threshold; PWL, paw withdrawal latency; NC, negative control.

cord tissue and microglia time-dependently. To further explore the roles of miR-154-5p in CCI rats, lentiviral vector LV-miR-154-5p was constructed and injected into rats. The levels of miR-154-5p, COX2, TNF- $\alpha$ and IL-6 were detected. The results demonstrated that miR-154-5p was significantly increased in LV-miR-154-5p and the levels of COX2, TNF- $\alpha$ and IL-6 were significantly decreased in LV-miR-154-5p group, suggesting that miR-154-5p overexpression inhibited inflammatory responses in $\mathrm{CCI}$ rats.

To further explore the detailed mechanism of miR-154-5p in inhibiting the inflammatory responses in CCI rats, target genes were analyzed by the TargetScan database and AQP9 was predicted to be a target gene of miR-154-5p. It was identified that the mRNA and protein levels of AQP9 in CCI rats were significantly increased in spinal cord tissue and microglia time-dependently. A wt-AQP9 luciferase reporter vector and a mut-AQP9 3'UTR luciferase reporter vector were constructed and the luciferase reporter gene assay was performed. The results demonstrated that miR-154-5p could directly bind with AQP9.

$\mathrm{SCI}$ is one of the most debilitating neurological conditions and most SCI patients remain in a chronic phase. As the specific molecular mechanism of neuropathic pain is unclear, there is still no effective intervention and treatment. A previous study demonstrated that MALAT1 has a protective neuroprotective effect through regulating with miR-204 (15). The present study demonstrated that the inhibition of MALAT1 in CCI rats could reduce neuropathic pain and neuroinflammation, 
suggesting that MALAT1 might be used as a new target for relieving neuropathic pain in humans and that it might provide a new intervention strategy for patients with SCI; however, this should be verified in human SCI patients.

Previous studies have demonstrated that the aquaporin family serve an important role in SCI $(35,36)$. AQP1/4/9 is expressed in a variety of cells such as astrocytes, neurons, and ependymal cells, and AQP1/4/9 in the spinal cord is involved in edema caused by SCI (35). AQP1 levels were significantly elevated in the SCI-induced injury site in rats, and AQP1 and mechanical ectopic pain were significantly decreased following melatonin treatment, suggesting that AQP1 may be associated with chronic neuropathic pain following SCI (36). As a member of the aquaporin family, AQP9 may also serve a role in neuropathic pain following SCI, but the specific function and mechanism of action are unknown. The present study identified that AQP9 expression was significantly upregulated in CCI rats and that neuropathic pain was attenuated following inhibition of AQP9, which was regulated through MALAT1/miR-154-5p axis. This suggested that the aquaporin family might be an important reference intervention target for the treatment of neuropathic pain in humans, but this also needs verification in human SCI patients.

Taken together, the results of the present study suggested that MALAT1/miR-154-5p/AQP9 served an important role in neuropathic pain and that the downregulation of MALAT1 attenuates neuropathic pain and inhibits neuroinflammation. In addition, MALAT1 inhibited neuropathic pain in rats by targeting the binding of miR-154-5p to inhibit AQP9 expression, which provided a potential prognostic marker and a potential target for neuropathic pain.

\section{Acknowledgements}

Not applicable.

\section{Funding}

No funding was received.

\section{Availability of data and materials}

The datasets used and/or analyzed during the current study are available from the corresponding author on reasonable request.

\section{Authors' contributions}

HD designed the present study. JW and CW performed the experiments. JW analyzed the data. All authors have read and approved the final manuscript.

\section{Ethics approval and consent to participate}

The present study was carried out in strict accordance with the requirements in the Guide for the Care and Use of Laboratory Animals of the National Institutes of Health. The experiments were carried out strictly in line with the requirements of the International Association for the Study of Pain (IASP). The present study was approved by the ethics committee of Lishui Municipal Central Hospital.

\section{Patient consent for publication}

Not applicable.

\section{Competing interests}

The authors declare that they have no competing interests.

\section{References}

1. Thomson S: Failed back surgery syndrome-definition, epidemiology and demographics. Br J Pain 7: 56-59, 2013.

2. Thomson S and Jacques L: Demographic characteristics of patients with severe neuropathic pain secondary to failed back surgery syndrome. Pain Pract 9: 206-215, 2009.

3. Liu XG, Pang RP, Zhou LJ, Wei XH and Zang Y: Neuropathic pain: Sensory nerve injury or motor nerve injury? Adv Exp Med Biol 904: 59-75, 2016.

4. Yalbuzdag SA, Erol AM, Sengul I, Celik C, Solum S, Adilay HU and Gungor B: Temperament and character profile in failed back surgery syndrome: A cross-sectional clinical study. Turk Neurosurg 26: 912-917, 2016.

5. Ellis A and Bennett DL: Neuroinflammation and the generation of neuropathic pain. Br J Anaesth 111: 26-37, 2013.

6. Yadav R and Weng HR: EZH2 regulates spinal neuroinflammation in rats with neuropathic pain. Neuroscience 349: 106-117, 2017.

7. Hauptman N and Glavac D: MicroRNAs and long non-coding RNAs: Prospects in diagnostics and therapy of cancer. Radiol Oncol 47: 311-318, 2013.

8. Morris KV and Mattick JS: The rise of regulatory RNA. Nat Rev Genet 15: 423-437, 2014.

9. Gu S, Xie R, Liu X, Shou J, Gu W and Che X: Long coding RNA XIST contributes to neuronal apoptosis through the downregulation of AKT phosphorylation and is negatively regulated by miR-494 in rat spinal cord injury. Int J Mol Sci 18: E732, 2017.

10. Li ZX, Zhu QN, Zhang HB, Hu Y, Wang G and Zhu YS: MALAT1: A potential biomarker in cancer. Cancer Manag Res 10: 6757-6768, 2018.

11. Sun Y and Ma L: New insights into long non-coding RNA MALAT1 in cancer and metastasis. Cancers (Basel) 11: E216, 2019.

12. Mei H, Liu Y, Zhou Q, Hu K and Liu Y: Long noncoding RNA MALAT1 acts as a potential biomarker in cancer diagnosis and detection: A meta-analysis. Biomark Med 13: 45-54, 2019.

13. Xia C, Liang S, He Z, Zhu X, Chen R and Chen J: Metformin, a first-line drug for type 2 diabetes mellitus, disrupts the MALAT1/miR-142-3p sponge to decrease invasion and migration in cervical cancer cells. Eur J Pharmacol 830: 59-67, 2018.

14. Zhang Y, Wu H, Wang F, Ye M, Zhu H and Bu S: Long non-coding RNA MALAT1 expression in patients with gestational diabetes mellitus. Int J Gynaecol Obstet 140: 164-169, 2018.

15. Qiao Y, Peng C, Li J, Wu D and Wang X: LncRNA MALAT1 is neuroprotective in a rat model of spinal cord ischemiareperfusion injury through miR-204 regulation. Curr Neurovasc Res 15: 211-219, 2018

16. Xie X, Ma L, Xi K, Zhang W and Fan D: MicroRNA-183 suppresses neuropathic pain and expression of AMPA receptors by targeting mTOR/VEGF signaling pathway. Cell Physiol Biochem 41: 181-192, 2017.

17. Yang Z, Xu J, Zhu R and Liu L: Down-regulation of miRNA-128 contributes to neuropathic pain following spinal cord injury via activation of P38. Med Sci Monit 23: 405-411, 2017.

18. Zheng Y, Zhu C, Ma L, Shao P, Qin C, Li P, Cao Q, Ju X, Cheng G, Zhu Q, et al: miRNA-154-5p inhibits proliferation, migration and invasion by targeting E2F5 in prostate cancer cell lines. Urol Int 98: 102-110, 2017.

19. Wang X, Sun S, Tong X, Ma Q, Di H, Fu T, Sun Z, Cai Y, Fan W, Wu Q, et al: MiRNA-154-5p inhibits cell proliferation and metastasis by targeting PIWIL1 in glioblastoma. Brain Res 1676: 69-76, 2017.

20. Damiano A, Zotta E, Goldstein J, Reisin I and Ibarra C: Water channel proteins AQP3 and AQP9 are present in syncytiotrophoblast of human term placenta. Placenta 22: 776-781, 2001.

21. Jelen S, Parm Ulhøi B, Larsen A, Frøkiær J, Nielsen S and Rützler M: AQP9 expression in glioblastoma multiforme tumors is limited to a small population of astrocytic cells and CD15(+)/CalB(+) leukocytes. PLoS One 8: e75764, 2013. 
22. Lv Y, Huang Q, Dai W, Jie Y, Yu G, Fan X, Wu A and Miao Q AQP9 promotes astrocytoma cell invasion and motility via the AKT pathway. Oncol Lett 16: 6059-6064, 2018.

23. Huang D, Feng X, Liu Y, Deng Y, Chen H, Chen D, Fang L, Cai Y, Liu H, Wang L, et al: AQP9-induced cell cycle arrest is associated with RAS activation and improves chemotherapy treatment efficacy in colorectal cancer. Cell Death Dis 8: e2894, 2017.

24. Chung JM, Kim HK and Chung K: Segmental spinal nerve ligation model of neuropathic pain. Methods Mol Med 99: 35-45, 2004.

25. National Research Council (US) Committee for the Update of the Guide for the Care and Use of Laboratory Animals: Guide for the Care and Use of Laboratory Animals. National Academies Press (US), Washington, DC, 2011.

26. Breivik H: International Association for the study of pain: Update on WHO-IASP activities. J Pain Symptom Manage 24: 97-101, 2002.

27. Beltramo M, Bernardini N, Bertorelli R, Campanella M, Nicolussi E, Fredduzzi S and Reggiani A: CB2 receptor-mediated antihyperalgesia: Possible direct involvement of neural mechanisms. Eur J Neurosci 23: 1530-1538, 2006

28. Livak KJ and Schmittgen TD: Analysis of relative gene expression data using real-time quantitative PCR and the 2(-Delta Delta C(T)) method. Methods 25: 402-408, 2001.

29. Li JH, Liu S, Zhou H, Qu LH and Yang JH: StarBase v2.0: Decoding miRNA-ceRNA, miRNA-ncRNA and protein-RNA interaction networks from large-scale CLIP-Seq data. Nucleic Acids Res 42: D92-D97, 2014.
30. Torrance N, Smith BH, Watson MC and Bennett MI: Medication and treatment use in primary care patients with chronic pain of predominantly neuropathic origin. Fam Pract 24: 481-485, 2007.

31. Torrance N, Smith BH, Bennett MI and Lee A: The epidemiology of chronic pain of predominantly neuropathic origin. Results from a general population survey. J Pain 7: 281-289, 2006.

32. Qiao W, Cao N and Yang L: MicroRNA-154 inhibits the growth and metastasis of gastric cancer cells by directly targeting MTDH. Oncol Lett 14: 3268-3274, 2017.

33. Xin C, Zhang $\mathrm{H}$ and Liu Z: miR-154 suppresses colorectal cancer cell growth and motility by targeting TLR2J. Mol Cell Biochem 387: 271-277, 2014.

34. Pang X, Huang K, Zhang Q, Zhang Y and Niu J: miR-154 targeting ZEB2 in hepatocellular carcinoma functions as a potential tumor suppressor. Oncol Rep 34: 3272-3279, 2015.

35. Halsey AM, Conner AC, Bill RM, Logan A and Ahmed Z: Aquaporins and their regulation after spinal cord injury. Cells 7: E174, 2018.

36. Nesic O, Lee J, Unabia GC, Johnson K, Ye Z, Vergara L, Hulsebosch CE and Perez-Polo JR: Aquaporin 1-a novel player in spinal cord injury. J Neurochem 105: 628-640, 2008.

(i) () This work is licensed under a Creative Commons Attribution-NonCommercial-NoDerivatives 4.0 International (CC BY-NC-ND 4.0) License. 